\title{
The Eighth International Workshop on Planning and Scheduling for Space (IWPSS)
}

\author{
Robert Morris and Steve Chien
}

The Eighth International Workshop on Planning and Scheduling for Space (IWPSS 2013) was held on March 25-26, 2013, at the NASA Ames Research Center, Moffett Field, California. This was the eighth in a regular series that started in 1997.

$\mathrm{T}$ The Workshop on Planning and Scheduling for Space (IWPSS) focuses on the technical challenges and opportunities facing the AI planning and scheduling community when addressing the needs of a wide range of space-based applications, from mission operations to autonomy in space exploration vehicles. There have been eight workshops in the series. At this year's workshop held March 25-26, 2013, at the NASA Ames Research Center, Moffett Field, California, there were 26 technical papers and two invited talks on a wide range of topic areas relating to planning and scheduling, including: techniques and algorithms, applications to space or aerospace, planning, scheduling, plan execution, knowledge acquisition for planning and scheduling systems, embedded planning and execution systems, and other general topic areas. Applications included International Space Station payloads, space mission operations, human space flight, space observatories, planning for communications, airborne operations, and Earth observation scheduling. International researchers from space agencies, academia, and industry from Europe, America, Canada, and South America participated.

The two invited talks illustrated both the diverse applications of planning and scheduling technologies for space, as well as the degree to which these technologies have been successfully infused into space systems. Steve Chien from JPL presented a talk titled "Using Space, Air, Marine, and Ground Assets for Disaster Response and Environmental Monitoring." He described how space, air, Inin-situ, and marine assets have been integrated into sensor webs to enable detection, tracking, and response to a wide range of terrestrial phenomena. These techniques have been used to track flooding, cryosphere, volcanism, oceanographic events, and many other events of humanitarian, scientific, and environmental importance. These Sensorwebs sensor webs use artificial intelligence techniques such as machine learning, automated planning, and automated data analysis.

The second invited talk, by Jeremy Frank of NASA/Ames Research Center, was titled "Autonomous Mission Operations," and described NASA's Advanced Exploration Systems $\mathrm{Au}$ tonomous Mission Operations (AMO) project. This project conducted an empirical investigation of the impact of communication time delay in deep space on today's mission operations, and of the effect of using mission support tools designed to mitigate timedelay delay-related impacts. The project used NASA's Deep Space Habitat (DSH), an analog spacecraft habitat, for the simulation, and a number of scenarios covering a range of activities were applied.

In addition to the two full days of technical talks, there were demonstrations of six planning and scheduling systems in various stages of deployment. Copies of papers and slides are available at robotics.estec.esa.int/IWPSS.

The next IWPSS workshop will be held in 2015 at a location to be determined.

Robert Morris is a senior researcher in the Planning and Scheduling group at NASA Ames Research Center. His research interests include constraint-based approaches to optimization applied to problems in planning and scheduling.

Steve Chien is the technical group supervisor of the Artificial Intelligence Group and a principal computer scientist in the Mission Planning and Execution Section at the Jet Propulsion Laboratory, California Institute of Technology where he leads efforts in automated planning and scheduling for space exploration. Chien is also an adjunct associate professor with the Department of Computer Science of the University of Southern California. 